\title{
Structural Stability of Transparent Conducting Films Assembled from Length Purified Single-Wall Carbon Nanotubes
}

\author{
John M. Harris, ${ }^{\dagger}$ Ganjigunte R. S. Iyer, ${ }^{\dagger}$ Daneesh O. Simien, ${ }^{\ddagger}$ Jeffrey A. Fagan, ${ }^{\S}$ Ji Yeon Huh, ${ }^{\S}$
}

Jun Young Chung, ${ }^{\S}$ Steven D. Hudson, ${ }^{\S}$ Jan Obrzut, ${ }^{\S}$ Jack F. Douglas,

Christopher M. Stafford, ${ }^{\S}$ and Erik K. Hobbie ${ }^{*,+}$

${ }^{+}$Department of Physics, Department of Coatings and Polymeric Materials, North Dakota State University, Fargo, North Dakota 58108,
United States
${ }^{\ddagger}$ Department of Mechanical and Aerospace Engineering, West Virginia University, Morgantown, West Virginia 26506, United States
${ }^{\$}$ Polymers Division, National Institute of Standards and Technology, Gaithersburg Maryland 20899, United States

ABSTRACT:
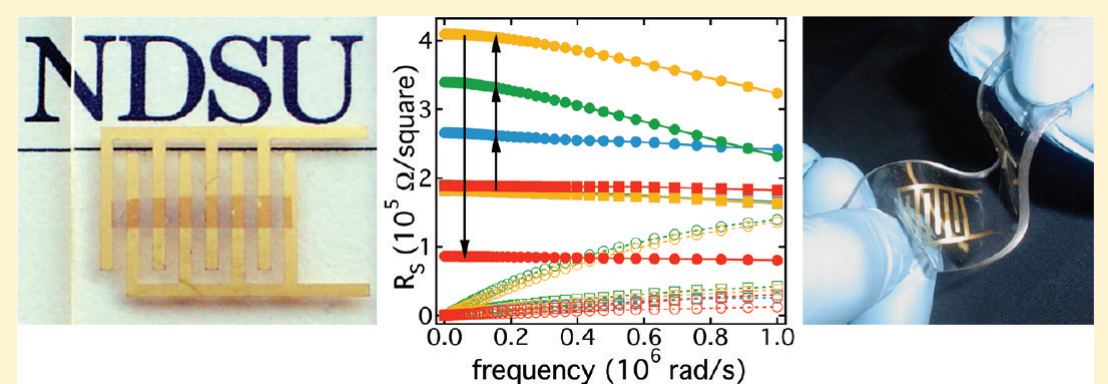

Single-wall carbon nanotube (SWCNT) films show significant promise for transparent electronics applications that demand mechanical flexibility, but durability remains an outstanding issue. In this work, thin membranes of length purified single-wall carbon nanotubes (SWCNTs) are uniaxially and isotropically compressed by depositing them on prestrained polymer substrates. Upon release of the strain, the topography, microstructure, and conductivity of the films are characterized using a combination of optical/fluorescence microscopy, light scattering, force microscopy, electron microscopy, and impedance spectroscopy. Above a critical surface mass density, films assembled from nanotubes of well-defined length exhibit a strongly nonlinear mechanical response. The measured strain dependence reveals a dramatic softening that occurs through an alignment of the SWCNTs normal to the direction of prestrain, which at small strains is also apparent as an anisotropic increase in sheet resistance along the same direction. At higher strains, the membrane conductivities increase due to a compression-induced restoration of conductive pathways. Our measurements reveal the fundamental mode of elasto-plastic deformation in these films and suggest how it might be suppressed.

\section{INTRODUCTION}

In the past two decades, single-wall carbon nanotubes (SWCNTs) have received considerable attention due to their outstanding mechanical, optical, and electronic properties, and a vast amount of research has been devoted to the characterization of these attributes ${ }^{1}$ and the potential applications they suggest. ${ }^{2}$ Promising applications are rapidly emerging in such areas as high performance composites, ${ }^{3}$ thermoelectric materials, ${ }^{4}$ and conducting polymer composites. ${ }^{5}$ Thin SWCNT films, in particular, show exceptional promise for applications that require transparent coatings with superior mechanical, electronic, and optical qualities. $^{6-9}$ The natural tendency for SWCNTs to form flexible, transparent networks with high electrical conductivity at remarkably low surface coverage is a direct consequence of the magnitude of the typical SWCNT aspect ratio, ${ }^{10}$ suggesting that nanotube length is a critical factor in dictating the physical properties of such membranes.
The electronic and optical properties of SWCNTs are determined by their electronic band structure, ${ }^{1}$ which is specified by the chiral vector $(n, m)$ characterizing the symmetry of rolling a two-dimensional graphene sheet into a hollow tube of diameter a. All existing synthetic techniques therefore produce raw material that contains a distribution of electronic types, ranging from semiconducting to metallic, as well as a broad range of lengths, from $10 \mathrm{~nm}$ up to hundreds of micrometers. Since scalable processes for purifying lab-grade quantities of SWCNTs have only recently been formulated, ${ }^{11-13}$ thin films of exemplary purified materials have just now become readily available for fundamental research, pointing toward a number of promising applications. Thin SWCNT membranes have recently emerged,

Received: January 10, 2011

Revised: January 23, 2011

Published: February 22, 2011 

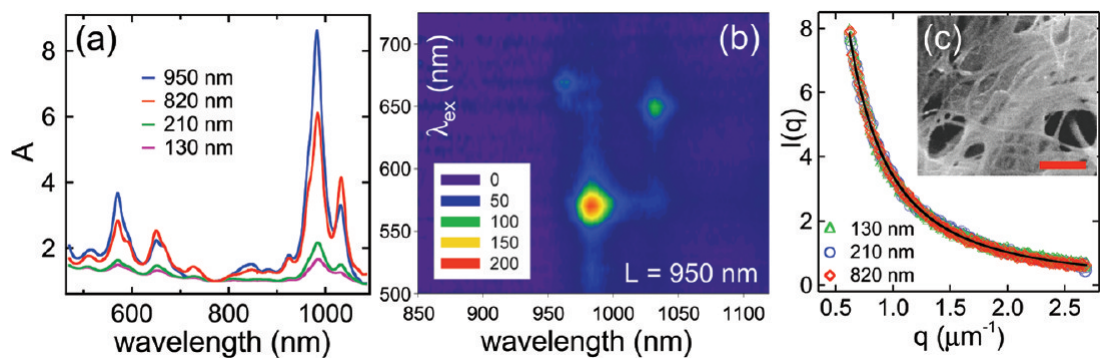

Figure 1. (a) Optical absorption spectra of the four different length fractions. (b) Typical near-infrared (NIR) fluorescence profile in the plane of emission and excitation $(L=950 \mathrm{~nm})$. (c) Small-angle light scattering profiles from unstrained dried membranes $\left(L=130 \mathrm{~nm}, c=3.5 \mu \mathrm{g} / \mathrm{cm}^{2} ; L=\right.$ $\left.210 \mathrm{~nm}, c=1.7 \mu \mathrm{g} / \mathrm{cm}^{2} ; L=820 \mathrm{~nm}, c=0.3 \mu \mathrm{g} / \mathrm{cm}^{2}\right)$ deposited on quartz, where the solid curve is a power law-fit to $q^{-1.72}$. The inset shows a scanningelectron microscopy (SEM) image of the porous SWCNT assembly in a dry membrane $\left(L=820 \mathrm{~nm}, c=0.02 \mu \mathrm{g} \mathrm{cm}^{-2}, \mathrm{scale}^{2}=100 \mathrm{~nm}\right)$.

for example, as a possible replacement for indium tin oxide (ITO) in photovoltaic devices ${ }^{14}$ and as potential electrode components in chemical fuel cells. ${ }^{15}$ As nanoporous two-dimensional rigid-rod networks, these structures also represent an intriguing class of mesostructured membrane with novel and potentially complex deformation mechanics. ${ }^{16-19}$

Commercial transparent conductive coatings like ITO tend to be brittle, and mechanical flexibility is often cited as a desirable characteristic in the next generation of applications. Given the outstanding intrinsic mechanical properties of SWCNTs, one can anticipate the added benefit that films of purified materials might offer in the area of mechanical performance, beyond the usually quoted optical and electronic benchmarks. The bending mechanics of individual SWCNTs on flexible polymer substrates have recently been investigated by measuring the amplitude and wavelength associated with periodic nanotube buckling under a compressive strain. ${ }^{20}$ Applying a continuum-mechanical model to such data yields a nanotube Young's modulus of around 1.3 $\mathrm{TPa}$ in reasonable agreement with predictions. ${ }^{21-26}$ Analogous mechanical studies of quasi-two-dimensional (2D) percolated SWCNT networks are much less developed. Mixed-length and mixed-type SWCNTs have been chemically treated, for example, to create strong interfacial adhesion on polydimethylsiloxane (PDMS) substrates, ${ }^{27}$ but chemical functionalization can have a significantly adverse effect on intrinsic nanotube properties ${ }^{28}$ despite the improved mechanical behavior that chemical grafting can induce.

In this contribution, we use a variety of characterization techniques to measure the mechanical and electronic response of thin membranes assembled from unfunctionalized length-purified SWCNTs on polymer substrates, and we correlate the mechanical behavior with changes in the electronic transport properties of the films. Our measurements reveal a material that is appropriately stiff under very small deformation, but which wrinkles and softens dramatically at finite strains. Correspondingly, this mechanical softening is initially apparent as an anisotropic decrease in film conductivity, but with a subsequent anisotropic increase at sufficiently high strains. In a recent Letter ${ }^{29}$ we modified a theoretical model originally developed by Balberg ${ }^{30}$ to link this type of strain softening to an upward shift in percolation threshold triggered by nonaffine strain-induced nanotube alignment. Here, we extend this analysis to measurements of film conductivity and we demonstrate the critical role of compliance at the electrode-membrane interface, a requirement that relates directly to the relatively small yield strains of the neat SWCNT films in question and puts limits on electrode thickness.

\section{RESULTS AND DISCUSSION}

Details related to nanotube purification, membrane production/transfer, and the techniques used to characterize the films can be found in the Methods and Materials. Surfactant-stabilized aqueous suspensions with mean SWCNT lengths $L=(950,820$, 210, and 130) nm were produced using density-gradient ultracentrifugation. ${ }^{13,31}$ The optical absorption spectra of the starting suspensions are shown in Figure 1a. Although of mixed electronic type, the SWCNTs have a natural enrichment in the $(6,5)$ semiconducting species by virtue of the synthetic scheme used to produce the raw materials. $^{32,33}$ The relatively small band gap leads to a photoluminescence in the near-infrared (NIR), which we exploit here to image the membranes with fluorescence microscopy. ${ }^{34}$ The thin $(10-100 \mathrm{~nm})$ SWCNT films are mesoporous (inset, Figure 1c) with a mass-fractal structure that can be measured in reciprocal space with small-angle light scattering (SALS); $I(q) \propto q^{-D}$ where $q$ is the scattered wave-vector (Figure 1c). The fractal dimension $D$ is insensitive to changes in $L$ and surface mass density, $c$, which is consistent with what has been observed in the high-aspect-ratio limit $(L / a \rightarrow \infty)$ for the diffusion-limited cluster aggregation of rodlike colloids. ${ }^{35}$ Somewhat intuitively, the influence of primary particle size diminishes once the nanotubes assemble into a network.

The wrinkling pattern that develops upon release of the strain reflects the disordered structure of the membranes, as shown in Figure $2 \mathrm{a}-\mathrm{d}$. In contrast to a pure harmonic, the deformation is hierarchical with an average length scale that decreases strongly with strain, which we identify as the wrinkling wavelength. The reciprocal-space structure factor (Figure $2 \mathrm{f}$ ) is analogous to that exhibited by a variety of complex materials under strain or flow, including a range of nanoparticle-polymer composites and complex fluids. $^{36}$ The morphology diagram (Figure $2 \mathrm{~g}$ ) shows three distinct states; unpercolated (region I, as determined by electrical impedance spectroscopy), percolated but unwrinkled up to $20 \%$ prestrain (region II, as determined by impedance spectroscopy and microscopy), and percolated with wrinkles (region III). The phase boundaries scale inversely with length, as predicted theoretically, ${ }^{29}$ and the three states can be distinctly resolved with NIR fluorescence microscopy near a membrane edge with multiple folds (Figure 2h).

The wrinkles seen in optical microscopy contain smaller folds that can be further resolved with force microscopy (AFM, Figure 3a,b) and scanning-electron microscopy (SEM, Figure 3c,d). Such creases are consistent with good adhesion of the SWCNT membrane to the PDMS; delamination of the film from the substrate leads to large regions of significant relief. At still smaller scales, we 


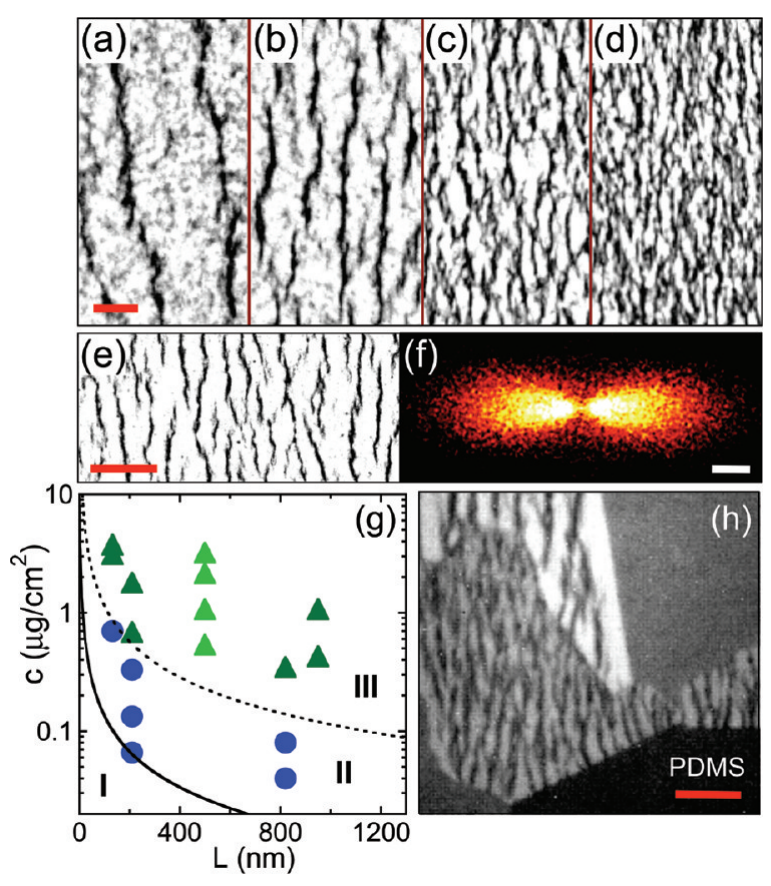

Figure 2. Reflection optical micrographs of SWCNT membrane wrinkling ( $\left.L=130 \mathrm{~nm}, c=3.5 \mu \mathrm{g} / \mathrm{cm}^{2}\right)$ at prestrains of (a) $2.5 \%$, (b) $5 \%$, (c) $10 \%$, and (d) $20 \%$. The scale bar is $5 \mu \mathrm{m}$. (e) Reflection optical micrographs of wrinkling $\left(L=130 \mathrm{~nm}, c=3.5 \mu \mathrm{g} / \mathrm{cm}^{2}\right)$ at $2.5 \%$ strain $($ scale $=$ $20 \mu \mathrm{m}$ ) and (f) the corresponding numerical power spectrum from the FFT (scale $\left.=0.63 \mu \mathrm{m}^{-1}\right)$. (g) State diagram for wrinkling in the plane of $c$ and $L$. The lower solid line is a fit of the connectivity percolation threshold to inverse aspect ratio and the upper dashed line is the wrinkling threshold. The blue symbols denote membranes that remain flat up to $20 \%$ prestrain, and the green symbols denote membrane wrinkling by $2 \%$ prestrain, where the light green markers show a mixedlength (unsorted) response. (h) NIR fluorescent micrograph ( $L=$ $130 \mathrm{~nm}$, prestrain $=5 \%$ ) showing percolated, unwrinkled membranes (upper right, lower left) and the linear increase in wrinkling wavelength with thickness.

can visualize the strain-induced anisotropy directly with transmission electron microscopy (TEM, Figure $4 a-c$ ), where the most striking contrast comes from SWCNT bundles aligned normal to the prestrain. Such bundles become apparent in the membranes at thicknesses and strains where the films appear optically flat, as shown in Figure 4c. As detailed below, the film thickness $h$ is a critical parameter in the analysis. We use AFM to measure the mean step height, $\hbar$, at the film edge (Figure 5a) and we use NIR fluorescence microscopy to measure the same step in $\mathrm{PL}$ at larger length scales (Figure $5 \mathrm{~b}$ ), which provides a consistent measure of SWCNT mass concentration that we use to refine the AFM measurements. As expected, the data suggest a linear relationship between $\hbar$ and $c$ (Figure 5c), where the slope is identified as the inverse SWCNT mass density $\left[\rho_{\mathrm{s}}=0.7 \pm 0.3 \mathrm{~g} /\right.$ $\mathrm{cm}^{3}$ for the data shown here]. To obtain an effective "envelope" or maximal thickness $h$ appropriate to a continuum theory, we thus divide $\hbar$ by the SWCNT volume fraction in the membranes.

The anisotropy that develops at the microscale is also evident in the structure factor measured with static light scattering, as can be seen by comparing the digital structure factor computed from optical micrographs (Figure 6a) with the measured SALS pattern (Figure 6b). Projected along the strain direction $(x)$, the scattering peaks coincide (Figure 6c) but the width of the peak normal to the strain direction $(y)$ is much smaller in SALS
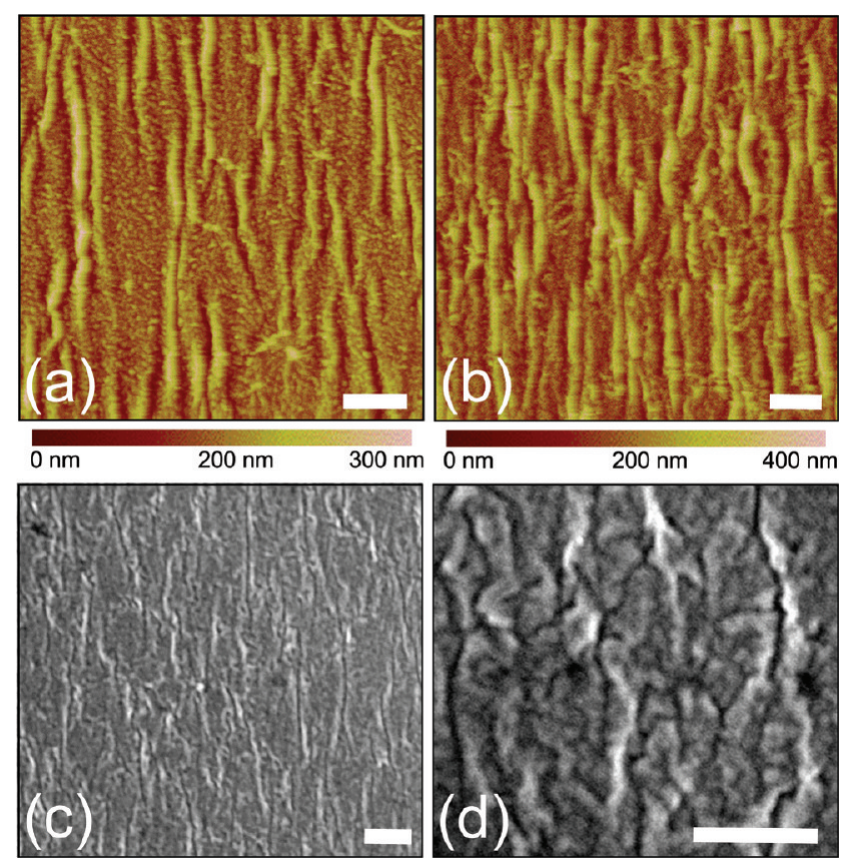

Figure 3. AFM images of the wrinkling topography for (a) $L=130 \mathrm{~nm}$, $c=3.5 \mu \mathrm{g} / \mathrm{cm}^{2}$, and a prestrain of $2.5 \%$ and (b) $L=130 \mathrm{~nm}, c=3.5 \mu \mathrm{g} /$ $\mathrm{cm}^{2}$, and a prestrain of $5 \%$, where the scale in each image is $5 \mu \mathrm{m}$. (c) and (d) SEM image reveals the complex topography of wrinkling at smaller length scales $\left(L=130 \mathrm{~nm}, c=3.5 \mu \mathrm{g} / \mathrm{cm}^{2}\right.$, prestrain $=5 \%$, scale bar $=$ $3 \mu \mathrm{m})$.

(Figure 6d). The peak position provides a measure of the characteristic wrinkling length equivalent to that obtained directly from real space micrographs (Figure 6e). In contrast, Cartesian projections of the pattern in Figure 6a provide a measure of the coherent dimensions, $\xi_{x}$ and $\xi_{y}$, associated with film topography at optical scales. The correlated spacing of these anisotropic structures gives rise to the scattering peaks, with the peak width providing a stronger measure of shape. The smaller width measured with SALS (Figure 6d) is consistent with the stronger underlying anisotropy revealed by TEM (Figure 6g), which corresponds to SWCNT bundles aligned normal to the prestrain.

The nonlinear buckling of thin stiff membranes on soft substrates is a long-standing problem in continuum mechanics. In the realm of nanotechnology, this approach has proven useful for determining thin-film elasticity where traditional methods of measurement are impossible. ${ }^{37}$ A common theme is the deposition of a uniform film of relatively high modulus onto a much thicker and softer substrate that has been stretched uniaxially to a specified prestrain $\varepsilon$. In the linear regime, the membrane is modeled as a buckled plate and the substrate as a Hookean solid. ${ }^{38} \mathrm{~A}$ homogeneous film of thickness $h$ and modulus $E_{\mathrm{f}}$ compressed on a softer substrate develops a sinusoidal wrinkling pattern of wavelength $\lambda_{0}=2 \pi h\left(\bar{E}_{\mathrm{f}} / 3 \bar{E}_{\mathrm{s}}\right)^{1 / 3}$ and amplitude $A_{0}=h\left(\varepsilon / \varepsilon_{\mathrm{c}}-1\right)^{1 / 2}$ above a critical strain $\varepsilon_{\mathrm{c}}={ }^{1} /{ }_{4}\left(3 \bar{E}_{\mathrm{s}} / \bar{E}_{\mathrm{f}}\right)^{2 / 3}$, where $\bar{E}_{\mathrm{i}}=E_{\mathrm{i}} /\left(1-v_{\mathrm{i}}{ }^{2}\right)$ is the plane-strain modulus defined in terms of Young's modulus $E_{\mathrm{i}}$ and Poisson ratio $v_{\mathrm{i}}$ of the film $(\mathrm{i}=\mathrm{f})$ or substrate $(\mathrm{i}=\mathrm{s})$. To extend this formalism to larger strains, the substrate is modeled as a neo-Hookean solid and $\lambda_{0}$ is scaled by a geometrical prefactor ${ }^{38}$ to give $\lambda=\lambda_{0}(1+\varepsilon)^{-1}(1+\zeta)^{-1 / 3}$ and $A=A_{0} /\left[(1+\varepsilon)^{1 / 2}(1+\right.$ $\left.\zeta)^{1 / 3}\right]$, where $\zeta=5 \varepsilon(1+\varepsilon) / 32$. The modulus and Poisson ratio of the PDMS substrate are determined independently (Materials 

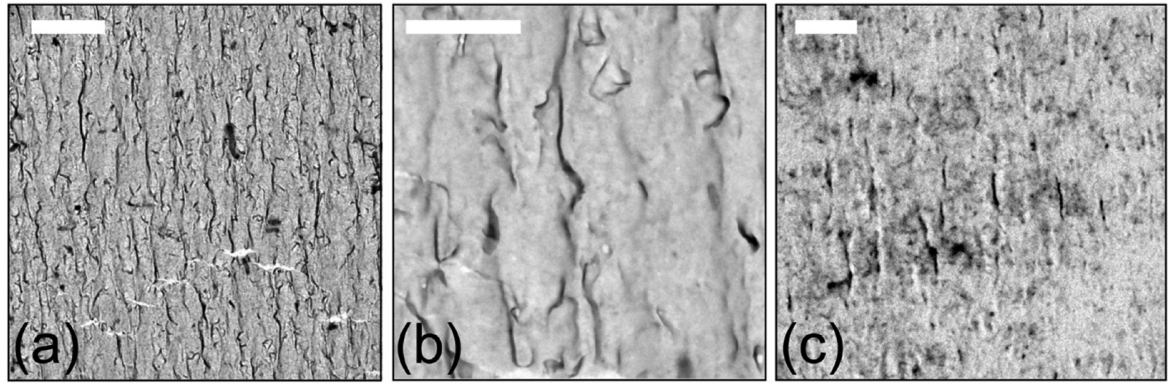

Figure 4. TEM images of membranes for (a) $L=130 \mathrm{~nm}, c=3.5 \mu \mathrm{g} / \mathrm{cm}^{2}$, and a prestrain of $20 \%$ (scale bar $\left.5 \mu \mathrm{m}\right),(\mathrm{b}) L=130 \mathrm{~nm}, c=3.5 \mu \mathrm{g} / \mathrm{cm}{ }^{2}$, and a prestrain of $20 \%$ (scale bar $2 \mu \mathrm{m}$ ), and (c) $L=130 \mathrm{~nm}, c=0.7 \mu \mathrm{g} / \mathrm{cm}^{2}$, and a prestrain of $20 \%$ (scale bar $1 \mu \mathrm{m}$ ). The sample in (c) resides on the boundary between percolated and wrinkled in the state diagram shown in Figure 2 and shows isolated regions of SWCNT alignment.
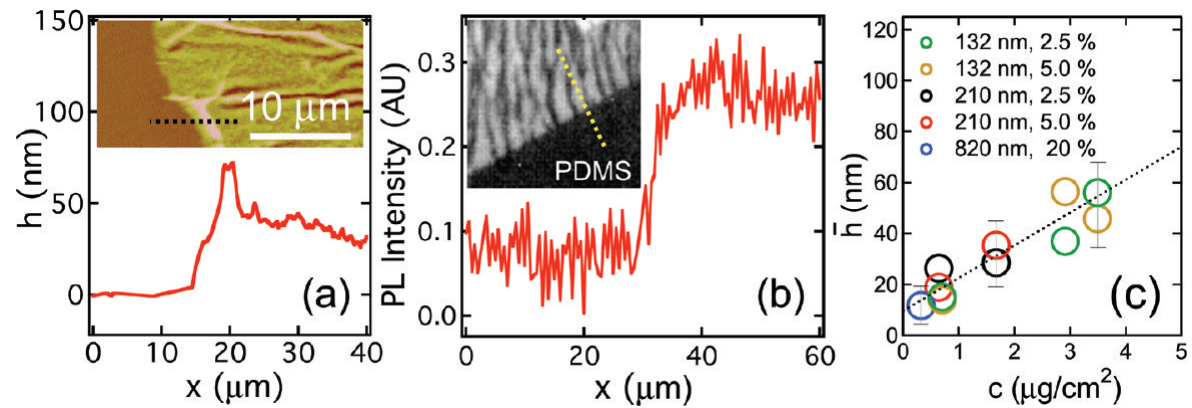

Figure 5. (a) Typical step-height profile (film thickness) measured with AFM and (b) measured with NIR fluorescence. (c) Mean membrane thickness as a function of surface concentration showing linear behavior for different SWCNT lengths.
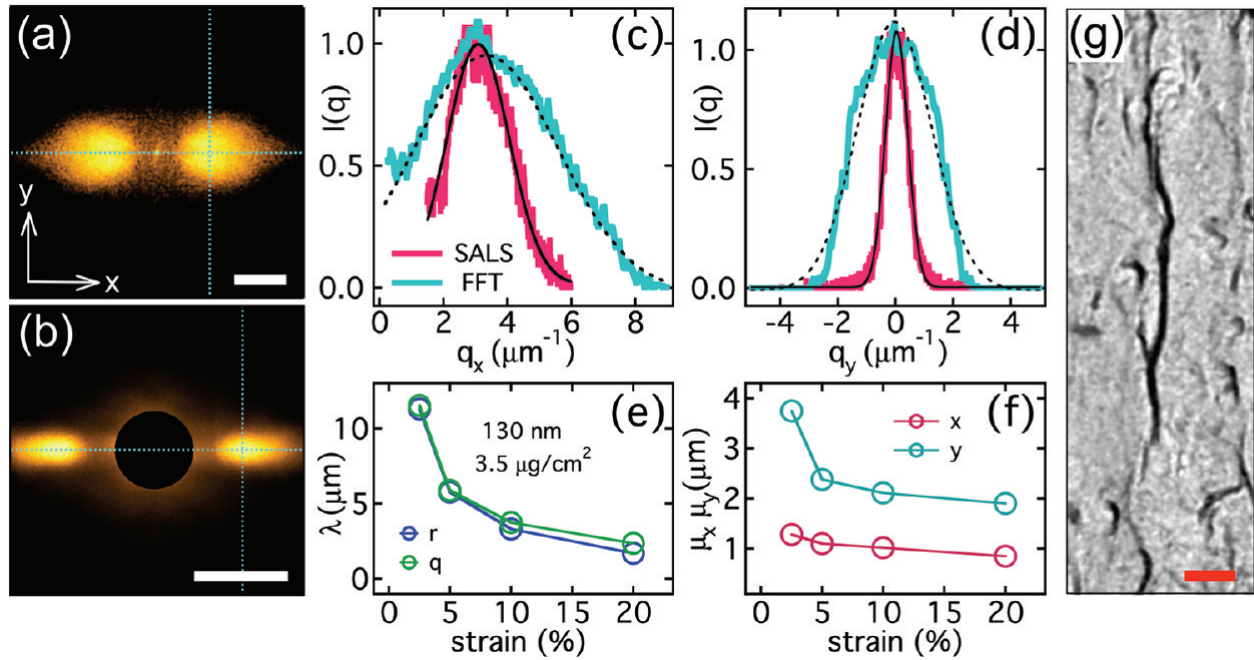

Figure 6. (a) Digital structure factor from an ensemble of optical micrographs and (b) the corresponding light-scattering pattern ( $10 \%$ prestrain, $L=$ $130 \mathrm{~nm}, c=2.9 \mu \mathrm{g} \mathrm{cm}^{-2}$, scale $\left.=3 \mu \mathrm{m}^{-1}\right)$. The compression of the two symmetric lobes along the $y$-axis, normal to the direction of prestrain suggests anisotropic bundling normal to the direction of prestrain. (c) Projected structure along the strain [horizontal dashed lines in (a) and (b)] with Gaussian fits and (d) projected structure normal to the strain [vertical dashed lines in (a) and (b)] with Gaussian fits. (e) Comparison of wrinkling wavelength determined in real space and reciprocal space. (f) Coherence lengths along and normal to the direction of strain. (g) Anisotropic bundling revealed by $\operatorname{TEM}($ scale $=200 \mathrm{~nm})$.

and Methods), and a Poisson ratio of 0.3 is assumed for the SWCNT films. ${ }^{39}$ Since $E_{\mathrm{f}} \propto\left(\lambda_{0} / h\right)^{3}$, it is critical to know the film thickness with precision and accuracy.

The optically determined characteristic length scale $(\lambda)$ decreases more strongly with prestrain than predicted by the above formalism, which we can explain if we allow the film modulus to be strain dependent. The fitted curves (Figure 7 and 8) are thus generalizations of the continuum model to allow for a strain dependent modulus; $E_{\mathrm{f}}(\varepsilon)=E_{\mathrm{f} 0}\left(1+\varepsilon / \varepsilon_{0}\right)^{-\delta}$, where $E_{\mathrm{fo}}$ is the modulus with respect to infinitesimal deformation and $\varepsilon_{0}$ is the yield strain. Our data are consistent with $\delta \approx 2.5$. As described previously, ${ }^{29}$ this expression gives an excellent representation of a more sophisticated model that relates this strain dependence to a shift in percolation 

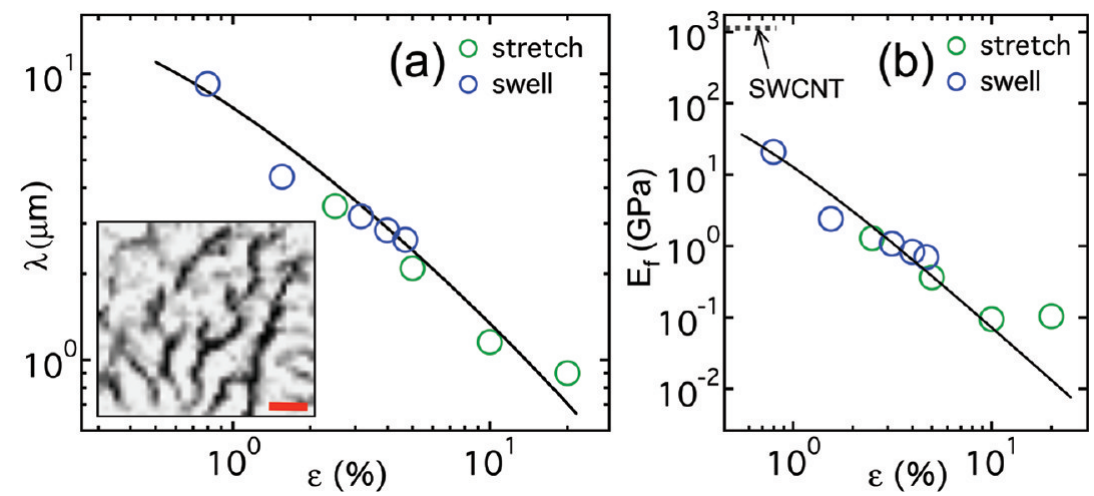

Figure 7. (a) Small and large-strain behavior of a SWCNT membrane $\left(L=950 \mathrm{~nm}, c=1 \mu \mathrm{g} \mathrm{cm}^{-2}\right)$, where the former are deduced from optical measurements during the slow drying of a preswelled PDMS substrate (blue markers). The inset shows typical topography at $4.7 \%$ strain $(\mathrm{scale}=4 \mu \mathrm{m})$. Wrinkling becomes apparent above a critical strain (vertical dashed line) comparable in magnitude to the yield strain, and the data match stretching data (green markers with fit) for the same membrane. (b) Film modulus as a function of strain deduced from the data in (a), with the typical modulus of an individual SWCNT indicated.
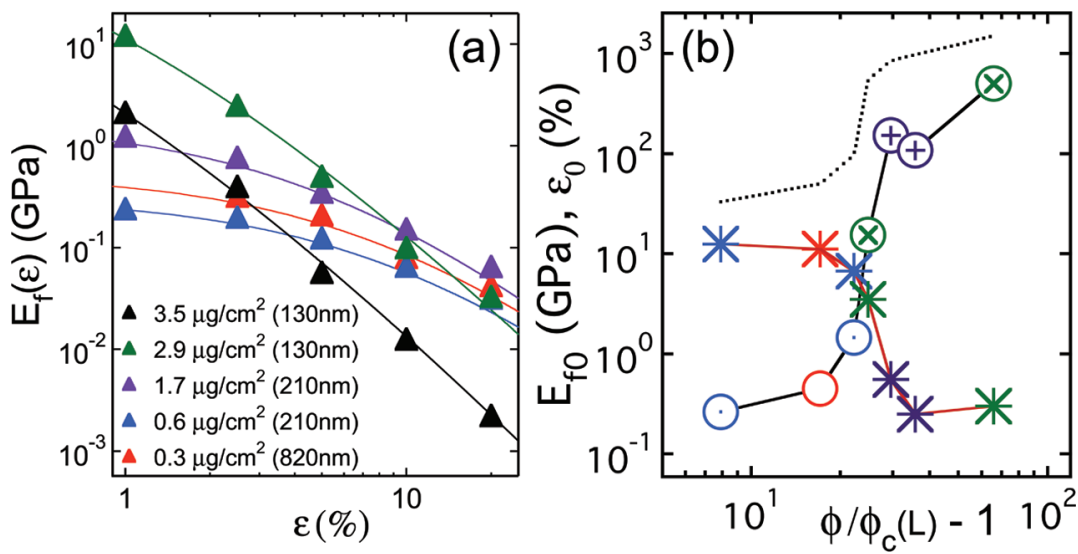

Figure 8. (a) Extracted modulus as a function of strain for different films. (b) Film modulus (circles) and yield strain (stars) as a function of reduced percolation depth for SWCNT lengths of $130 \mathrm{~nm}$ (cross), $210 \mathrm{~nm}$ (dot), $820 \mathrm{~nm}$ (open), and $950 \mathrm{~nm}$ (hatch). The dashed curve is the suggested yield stress in MPa.
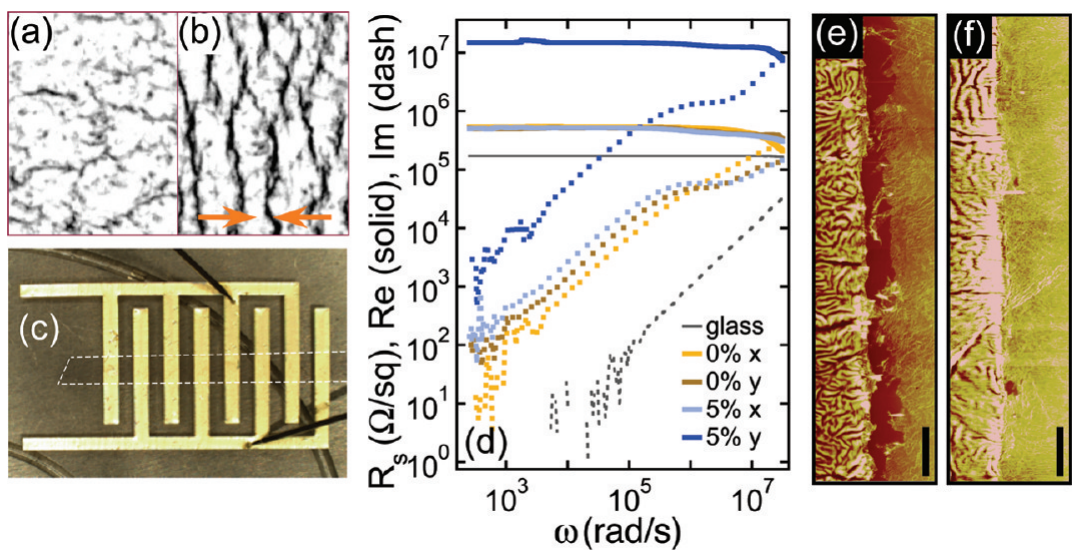

Figure 9. (a) Region of a membrane $\left(L=130 \mathrm{~nm}, c=3.5 \mu \mathrm{g} / \mathrm{cm}^{2}\right)$ before the release of a $5 \%$ prestrain and (b) the same region after release. (c) Electrode configuration used to measure the sheet resistance of the strained membranes, where the dashed white boundary delineates the SWCNT membrane. (d) Complex sheet resistance as a function of frequency for the membrane depicted in (a) and (b) deposited on glass, on unstrained PDMS, and on PDMS after the release of a 5\% prestrain. (e) Fractured gold (left) - SWCNT (right) interface ( $L=950 \mathrm{~nm}, c=1 \mu \mathrm{g} \mathrm{cm}^{-2}, 2.5 \%$ prestrain). The electrode is $200 \mathrm{~nm}$ thick ( $25 \mu \mathrm{m}$ scale). (f) Nearly intact gold-SWCNT interface for a piece of the same membrane at $15 \%$ prestrain with a $100 \mathrm{~nm}$ thick electrode $(25 \mu \mathrm{m}$ scale).

threshold triggered by nanotube alignment. The softening of the membranes has the effect of suppressing the increase in wrinkling amplitude with strain predicted by the continuum model. Strain softening in these complex solids is evidently an analog of the power-law shear thinning commonly observed in complex fluids.

Deep into the percolation regime, the extracted zero-strain modulus $E_{\mathrm{fo}}$ (Figure $8 \mathrm{~b}$ ) approaches a value of $500 \mathrm{GPa}$, still well 
below the 1.3 TPa modulus of an individual SWCNT. The results suggest that $L$ enters the problem most strongly through the percolation threshold $c_{0}(L)$, since data for different SWCNT lengths fall on the same curve when plotted against $c / c_{0}(L)-1$. Although the extrapolated modulus increases dramatically with increasing concentration, this increase is mirrored by a decrease in yield strain that puts limits on any mechanical enhancement. Here, the available measure of yield stress, $\varepsilon_{0} E_{\mathrm{f} 0}$, shows only a weak increase with concentration (Figure $8 b$ ). This is a common issue with carbon-nanotube composites and is why chemical functionalization is so often employed. The behavior described here is completely analogous to the type of yield-stress behavior exhibited by concentrated carbon-nanotube suspensions. ${ }^{40}$ Although the film modulus is extrapolated as a fitting parameter and is thus to some extent approximate, the reported trends with thickness and concentration are robust. The small yield strains are further supported by small-strain measurements that exploit a degree of PDMS swelling to introduce a controlled isotropic strain (Figure 7). Such measurements confirm the absence of a low-strain plateau in the wrinkling wavelength down to a critical strain that mirrors the yield strain in magnitude. Future efforts will focus on improving the mechanical behavior of these films through "passive" approaches, such as SWCNT purification by electronic type to improve the strength of van der Waals contact forces and the introduction of a second interpenetrating polymer, elastomer, or nanoparticle matrix.

An important and related question is how the softening affects electronic transport. In this regard, it is instructive to consider the evolution from hard to soft substrates, as depicted in Figure 9. The typical change in film morphology upon release of the prestrain is shown Figure 9a,b, where the texture in Figure 9a results from swelling of the PDMS substrate during transfer of the SWCNT film. The electrode geometry is shown in Figure 9c. The best transport properties are obtained for SWCNT films deposited on glass (Figure 9d), presumably because the strains associated with polymer substrate swelling/deswelling slightly disrupt the nanotube network. Important clues into the origin of the softening are provided by the decrease in electrical conductivity normal to the prestrain observed for modest strains, which indicates that conductive pathways are being anisotropically broken by the deformation of the film. This is likely the same breakdown of network contacts responsible for mechanical softening, and the connection between this developing anisotropy and the decrease in film stiffness and conductivity follows from the established observation that the alignment of extended particles reduces the percolation threshold, as recently demonstrated in sheared carbon nanotube suspensions ${ }^{41}$ and stretched SWCNT composites. ${ }^{42}$ Here, the orientation results from the strain imposed on the membrane through the combined contraction and Poisson expansion of the substrate. ${ }^{29}$

Although the Au electrodes are deposited on the samples after release of the prestrain, the fragility of the SWCNT membranes ultimately influences the nature of electrical contact with these electrodes, as shown in Figure 9e,f. Thicker electrodes $(200 \mathrm{~nm}$, Figure 9e) are less compliant to the substrate, which causes the SWCNT films to break at the interface in response to the routine handling associated with characterization. The situation can be substantially improved by using thinner electrodes $(100 \mathrm{~nm}$, Figure 9f). Accounting for breaks and defects in the films as measured through AFM and optical microscopy, reducing the electrode thickness from 200 to $100 \mathrm{~nm}$ results in a significant decrease in the sheet resistance of the membranes.
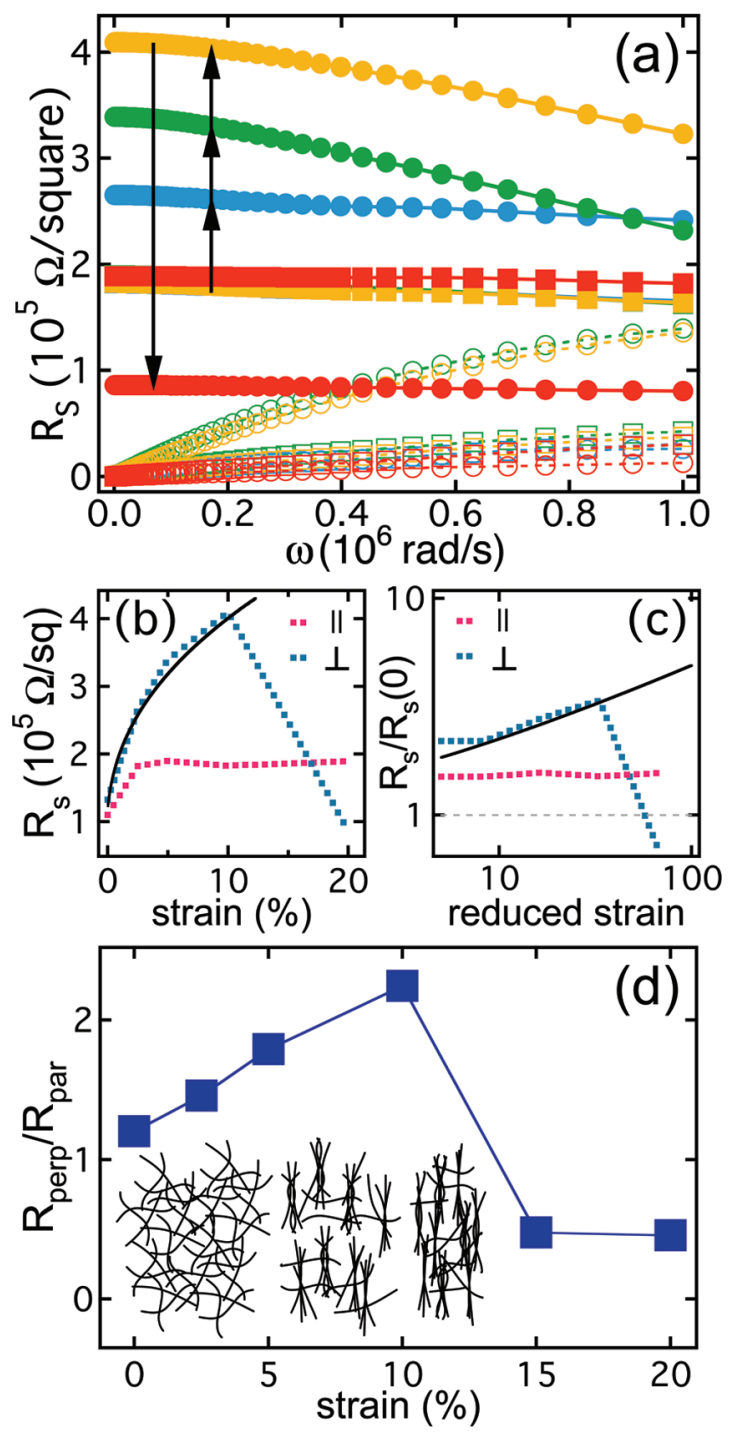

Figure 10. (a) Complex sheet resistance (real part, solid; imaginary part, open/dashed) as a function of frequency measured parallel (squares) and perpendicular (circles) to the direction of prestrain at $2.5 \%$ (blue), 5\% (green), 10\% (orange), and 20\% (red) strain. Data correspond to $L=950 \mathrm{~nm}$ and $c=1 \mu \mathrm{g} \mathrm{cm}^{-2}$. (b) Sheet resistance for the same sample as a function of strain normal to (blue) and along (red) the axis of prestrain. The black curve is the anticipated strain response based on the softening deduced from wrinkling and the measured percolation conductivity exponent. (c) Same data displayed as reduced sheet resistant as a function of reduced strain on a log-log scale. (d) Anisotropy in the sheet resistance as a function of strain. The inset illustrates the effect of strain on electrical connectivity in the membranes.

Finally, the evolution of sheet resistance with strain is examined in Figure 10. Well into the regime of equilibrium percolation, the conductivity normal to the prestrain initially decreases, as described above, with very little change along the direction of prestrain. Alignment leads to the breaking of network contacts normal to the prestrain, while compression maintains such contacts orthogonal to this. If the decrease is indeed linked to the softening of the membrane, then the two trends should scale accordingly. We previously measured ${ }^{43}$ the electrical percolation behavior of the same unstrained films on filter paper, assembled from identical SWCNT fractions, and found that the conductivity 
scaled as $\sigma \propto\left[c / c_{0}(L)-1\right]^{t}$ with $t=1.4$. Our model of strain softening ${ }^{29}$ also gives an expression for the strain-induced increase in percolation threshold; $c_{0}(\varepsilon) \approx c_{0}\left(1+\varepsilon / \varepsilon_{0}\right)^{\delta / \alpha}$ where $\alpha=10$ is the $2 \mathrm{D}$ percolation exponent for elasticity ${ }^{29}\left[E_{\mathrm{f}} \propto\left(c / c_{0}\right.\right.$ $\left.-1)^{\alpha}\right]$. These combine to give $R_{s}(\varepsilon) / R_{s}(0) \approx\left(1+\varepsilon / \varepsilon_{0}\right)^{\delta t / \alpha}$, where $R_{s}$ is the sheet resistance and there are no free parameters. For small strains, the data do indeed follow this trend (Figure 10a-c), but the tendency reverses at strains above $10 \%$ where the conductivity starts to increase. This can also be explained intuitively, since at sufficiently large strains the compression of the membrane will eventually bridge contacts between aligned bundles and restore the conductivity normal to the direction of prestrain (Figure 10d).

\section{- CONCLUSIONS}

Through the evolution of the wrinkling instability that emerges in compressed thin membranes of polymer supported SWCNTs as a function of film thickness and percolation depth, we demonstrate strain-softening in un-cross-linked length-purified SWCNT membranes. Network destruction due to nanotube alignment has been independently confirmed through conductivity measurements at small strains, but this trend is eventually overcome by the restoration of electrical contacts through compression. There is even the suggestion of a large-strain enhancement in conductivity that we speculate arises from improved charge transport along aligned SWCNT bundles that become laterally interconnected. These observations highlight an important distinction between electrical and elastic percolation that is also evident in the relative magnitude of the two critical exponents $t$ and $\alpha$. While the rigidity of the film can continue to improve with additional contacts, this is less true for conductivity. A few good contacts are sufficient for charge transport, and beyond this there is relatively weak improvement. Similarly, these contacts do not have to be mechanically rigid. In principle, a single interconnected path is sufficient to initiate charge flow, while many such pathways are required to establish isotropic rigidity.

An immediate implication of these observations is the use of these SWCNT films as electronic strain sensors. To a degree this is already possible and promising, as recently demonstrated using cross-linked CNT films. ${ }^{27}$ Substantial improvement can be made here by limiting the nonlinear plastic nature of the structural changes that arise through these types of deformation. ${ }^{29}$ The SWCNT bundling that we directly image here can be strongly irreversible, and improving the elastic response of these films will be the emphasis of future research. In the case of transparent conducting SWCNT films, incorporating a polymer into the nanotube network is a promising approach for improving mechanical performance, but the type of deformation imposed here is considerably more severe than what is usually considered. Our goal is to make the pristine SWCNT films as mechanically robust as possible by the metrics defined here, which will then offer a route to the engineering of conducting polymer nanocomposite films with exceptional optical and mechanical characteristics.

\section{MATERIALS AND METHODS}

Materials. SWCNTs grown by the cobalt - molybdenum catalyst (CoMoCat) process were purchased from Southwest Nanotechnologies (S-P95-02 grade, batch NI6-A001). They were dispersed at $1 \mathrm{mg}$ soot $/ \mathrm{mL}$ in a $2 \%$ by mass sodium deoxycholate (DOC) aqueous solution by tip sonication for $1.5 \mathrm{~h}$ in an ice bath at $1 \mathrm{~W} / \mathrm{mL}$ applied power. Purification was performed by centrifugation at $21 \times 10^{3} \mathrm{G}$ for $2 \mathrm{~h}$ to pellet the non-SWCNT impurities. Density layers were formed from varied concentrations of iodixanol-deoxycholate solutions. Separation was performed in a SW-32 rotor using a Beckman Coulter L80 XP ultracentrifuge at $1445 \mathrm{rad} / \mathrm{s}$ for $70 \mathrm{~h}$. Individual fractions were collected in $1.5 \mathrm{~mL}$ increments. From these, we selected four fractions, with $L$ as determined by AFM and TEM and further verified by the intensity of the resonant absorption peak at $984 \mathrm{~nm}$. The combined relative uncertainty in $L$ is $10 \%$ with a standard deviation of $20 \%$. Length fractions were dialyzed against $0.8 \%$ DOC solution to remove the remaining iodixanol and reduce the surfactant concentration.

Nanotubes were deposited on cellulose-ester filter paper of diameter $47 \mathrm{~mm}$ and mean pore size $0.05 \mu \mathrm{m}$ using vacuum filtration. A mixture of 2-propanol and deionized water (DI, 1:4 by volume) was used to condition the filter and DI water was used to rinse away surfactant. The surface density was controlled by quantifying the volume of fractionated suspension (of known concentration) using a micropipet and diluting the suspension with DI water to $2 \mathrm{~mL}$. The samples were dried at $50{ }^{\circ} \mathrm{C}$ for $24 \mathrm{~h}$ under a nitrogen atmosphere and the surface density for percolation determined through impedance spectroscopy as a function of surface coverage. The mass of SWCNT per unit area of the membrane $(c)$ was determined from the amount of SWCNT deposited onto a known area of filter paper. The concentration of SWCNT in the parent solution is determined from the nonresonant (baseline) optical absorption at $775 \mathrm{~nm}$. The extinction coefficient obtained $[2.1(\mathrm{~mL} / \mathrm{mm}) / \mathrm{mg}]$ is close to values in the literature and is consistent with the measured absorbance at 775 $\mathrm{nm}$ in the sonicated, precentrifuged suspension (for which the mass fraction is precisely known). The validity of the spectroscopic approach was further confirmed for both length polydisperse and length fractionated suspensions by directly weighing the amount of SWCNT deposited on filter paper membranes.

Substrates (PDMS, Sylgard 184; Dow Corning Co.) were prepared by hand mixing an oligomeric base and curing agent (10:1 by mass). The mixture was left at room temperature overnight to allow trapped air bubbles to escape and cured at $75^{\circ} \mathrm{C}$ for $2 \mathrm{~h}$. The cured PDMS substrates (thickness $\approx 1.5 \mathrm{~mm}$ ) were cut into $25 \mathrm{~mm} \times 75 \mathrm{~mm}$ strips. Each strain measurement corresponds to one PDMS substrate, with up to four SWCNT membranes on a substrate. The uniaxial strain was imposed on a strain gauge equipped with a micrometer ${ }^{44}$ and the rate of release was kept constant to control for variations in morphology with rate. The zero-strain state was defined in one of two ways. For measurements that could be performed on samples mounted in the gauge (such as scattering and optical microscopy), a small (ca. 5\%) reference strain was imposed prior to applying the prestrain and depositing the SWCNTs. The prestrain was then precisely defined by releasing the gauge back to this reference. This approach assumes the PDMS remains approximately linearelastic up to $30 \%$ strain, which we measured to be the case. Equivalently, transmission light microscopy of the mounted PDMS oriented at $45^{\circ}$ with respect to two crossed polarizers was used to monitor the transmitted intensity as a function of micrometer setting, and the stress-optical rule was then invoked to define the zero-strain position. The elastic modulus of the PDMS was measured to be $1.8 \mathrm{MPa}$ using a standard uniaxial tensile tester (Texture Analyzer, Model TA.XT2i; Texture Technologies) and a Poisson ratio of 0.48 was assumed. Using values 
other than 0.3 for the Poisson ratio of the SWCNT membranes had very little effect on the trends reported. To deduce the smallstrain behavior, PDMS substrates were maximally swelled in acetone before and during SWCNT membrane transfer, and the samples then slowly dried by allowing the acetone to evaporate. The strain was computed from changes in the relative position of obvious landmarks (defects) in the membranes apparent in image sequences collected during the drying of the samples. To compare with data from stretched films, the wavelength was rescaled to account for changes in substrate modulus with swelling based on the classical theory of rubber elasticity.

Small sections of each SWCNT membrane were deposited on PDMS (strained and unstrained), glass, and quartz by back dissolving the filter paper with acetone. The SWCNTs have a natural affinity for interfaces, even when they are stable by most metrics for colloidal dispersion. ${ }^{45}$ Substrates of PDMS completely immersed in acetone for $24 \mathrm{~h}$ swell, ${ }^{46}$ but the stretched films used here were exposed over a limited region of surface area for less than $30 \mathrm{~min}$ total. Residual strain from solvent swelling effects was estimated to be less than $2 \%$ and was orientated normal to the direction of the applied prestrain (Figure 9a). Consequently, any wrinkling due to swelling was relatively weak, was oriented at $90^{\circ}$ to the wrinkling front of interest, and was minimally discernible after release of the prestrain. The wrinkling wavelength is an ensemble average of values for several different regions of the same membrane obtained optically and with AFM.

Methods. Optical absorption spectroscopy was performed in transmission mode on a Perkin-Elmer Lambda $950 \mathrm{UV}-$ visNIR spectrophotometer at $185-1880 \mathrm{~nm}$. The incident light was circularly polarized and the instrument was corrected for both the dark current and background spectra. The reference beam was left unobstructed during the measurement with the subtraction of the appropriate reference sample performed during data reduction. Absorption spectra were recorded at $1 \mathrm{~nm}$ increments, with an instrument integration time of $0.2 \mathrm{~s}$ per increment, using a 1,2 , or $10 \mathrm{~mm}$ quartz cuvette, depending upon the extinction of a particular sample. Fluorescence characterization was performed on a JY-Horiba Nanolog-3 spectrofluorometer with a liquidnitrogen cooled InGaAs detector. Emission spectra were corrected for source spectral distribution, detector spectral response, and the absorbance of the filter used to restrict the scattered excitation light from the NIR monochromators and detector. The excitation wavelength was scanned in $5 \mathrm{~nm}$ increments using a $450 \mathrm{~W}$ xenon lamp through a $10 \mathrm{~nm}$ slit with emission collected at $90^{\circ}$ in either 1,2 , or $4 \mathrm{~nm}$ increments through a $10 \mathrm{~nm}$ slit.

The wrinkling morphology was characterized with SALS, optical/fluorescence microscopy, AFM, SEM, and TEM. Static light scattering measurements were performed with a $30 \mathrm{~mW}$ $\mathrm{He}-\mathrm{Ne}$ laser directed through the strained film, with the scattered light imaged on a screen equipped with a beam stop using a thermoelectrically cooled CCD. Optical microscopy was performed in both reflection and epi-illumination NIR fluorescence (with a liquid-nitrogen cooled InGaAs CCD) using 10$50 \times$ objectives. The local topography of the wrinkled membranes was measured with AFM in tapping mode. Scanning electron microscopy (SEM) measurements were performed using standard protocols on untreated membranes. Samples for TEM were prepared by shadowing the membranes with platinum/carbon (Pt/C) and then evaporating a thin film of carbon onto the shadowed membrane. A film of poly(acrylic acid) (PAA) was then cast onto this surface. After drying at $55^{\circ} \mathrm{C}$ overnight, the PAA was removed, causing the membrane and evaporated carbon to detach from the PDMS. The PAA was dissolved in water and the floating SWCNT membranes retrieved on copper grids. The thin $\mathrm{Pt} / \mathrm{C}$ coating provided only weak image contrast, the primary features arising from local variations in SWCNT density.

To measure sheet resistance, an interdigitated electrode pattern was sputtered onto the SWCNT membranes through a shadow mask. A 2-10 nm base layer of chromium was used to improve electrode contact and adhesion, followed by a 100-200 $\mathrm{nm}$ layer of gold, deposited over the chromium without breaking vacuum. Electrical measurements were analyzed in terms of complex impedance, yielding the impedance magnitude $\left(\left|Z^{*}\right|\right)$ and the corresponding phase angle $(\vartheta)$ as a function of frequency. The impedance was measured as a function of frequency between $10^{-2}$ and $10^{3} \mathrm{kHz}$ using a four-terminal fixture attached to an Agilent 4294A precision impedance analyzer, calibrated with an extension adapter to short, load, and open standards. Measurements were also performed on an Agilent B 1500A semiconductor device analyzer in both 2- and 4-probe geometries, calibrated against $1.01 \mu \mathrm{F}$ capacitor. The impedance was normalized to sheet resistance using the measured geometry of the electrode pattern and membrane to account for any breaks in the film. The combined relative experimental uncertainty is $4 \%$, and the experimental uncertainty of the phase angle measurements is $\pm 0.5^{\circ}$. For measurements on strained films, electrodes were deposited on two separate membranes prepared on the same PDMS substrate. One pair was oriented to measure film conductivity along the direction of prestrain and the other was rotated $90^{\circ}$ with respect to this.

\section{AUTHOR INFORMATION}

\section{Corresponding Author}

*E-mail: erik.hobbie@ndsu.edu.

\section{ACKNOWLEDGMENT}

Certain equipment, instruments, or materials are identified in this paper to adequately specify the experimental details. Such identification does not imply recommendation by the National Institute of Standards and Technology nor does it imply the materials are necessarily the best available for the purpose. E.K.H. acknowledges the support of the NSF through CMMI-0969155 (JH) and the DOE through DE-FG36-08GO88160 (SI). We also thank Thuy Chastek, Benjamin Forsythe, and Steven Ruckdashel for assistance with the measurements.

\section{REFERENCES}

(1) Saito, R.; Dresselhaus, G.; Dresselhaus, M. S., Physical Properties of Carbon Nanotubes; Imperial College Press: London, 1999.

(2) Baughman, R. H.; Zakhidov, A. A.; de Heer, W. A. Science 2002, 297, 787-792.

(3) Mauter, M. S.; Elimelech, M.; Osuji, C. O. ACS Nano 2010, 4, 6651-6658.

(4) Kim, D.; Kim, Y.; Choi, K.; Grunlan, J. C.; Yu, C. ACS Nano 2010, 4, 513-523.

(5) Hermant, M.-C.; van der Schoot, P.; Klumperman, B.; Koning, C. E. ACS Nano 2010, 4, 2242-2248.

(6) Cao, Q.; Rogers, J. A. Adv. Mater. 2008, 21, 29-53.

(7) Hu, L.; Hecht, D. S.; Grüner, G. Chem. Rev. 2010, 110, 5790-5844.

(8) Wu, Z. C.; Chen, Z. H.; Du, X.; Logan, J. M.; Sippel, J.; Nikolou, M.; Kamaras, K.; Reynolds, J. R.; Tanner, D. B.; Hebard, A. F.; et al. Science 2004, 305, 1273-1276. 
(9) De, S.; Lyons, P. E.; Sorrel, S.; Doherty, E. M.; King, P. J.; Blau, W. J.; Nirmalraj, P. N.; Boland, J. J.; Scardaci, V.; Joimel, J.; et al. ACS Nano 2009, 3, 714-720.

(10) De, S.; King, P. J.; Lyons, P. E.; Khan, U.; Coleman, J. N. ACS Nano 2010, 4, 7064-7072.

(11) Hersam, M. C. Nat. Nanotechnol. 2008, 3, 387-394.

(12) Tu, X.; Manohar, S.; Jagota, A.; Zheng, M. Nature 2009, $460,250-253$.

(13) Fagan, J. A.; Becker, M. L.; Chun, J.; Hobbie, E. K. Adv. Mater. 2008, 20, 1609-1613.

(14) Hu, L.; Hecht, D. S.; Grüner, G. Appl. Phys. Lett. 2009, 94, 081103.

(15) Landi, B. J.; Ganter, M. J.; Cress, C. D.; DiLeo, R. A.; Raffaelle, R. P. Energy Environ. Sci. 2009, 2, 638-654.

(16) Song, L.; Ci, L.; Lv, L.; Zhou, Z.; Yan, X.; Liu, D.; Yuan, H.; Gao, Y.; Wang, J.; Liu, L.; Zhao, X.; Zhang, Z.; Dou, X.; Zhou, W.; Wang, G.; Wang, C.; Xie, S. Adv. Mater. 2004, 16, 1529-1534.

(17) Baughman, R. H.; Cui, C.; Zakhidov, A. A.; Iqbal, Z.; Barisci, J. N.; Spinks, G. M.; Wallace, G. G.; Mazzoldi, A.; De Rossi, D.; Rinzler, A. G.; Jaschinski, O.; Roth, S.; Kertesz, M. Science 1999, 284, 1340-1344.

(18) Zhang, X. F.; Sreekumar, T. V.; Liu, T.; Kumar, S. J. Phys. Chem. B 2004, 108, 16435-16440.

(19) Volkov, A. N.; Zhigilei, L. V. ACS Nano 2010, 4, 6187-6195.

(20) Khang, D.-Y.; Xiao, J.; Kocabas, C.; MacLaren, S.; Banks, T.; Jiang, H.; Huang, Y. Y.; Rogers, J. A. Nano Lett. 2008, 8, 124-130.

(21) Li, C.; Chou, T.-W. Mech. Mater. 2004, 36, 1047-1055.

(22) Yakobson, B. I.; Brabec, C. J.; Bernholc, J. Phys. Rev. Lett. 1996, 76, 2511-2514.

(23) Yu, M.-F.; Files, B. S.; Arepalli, S.; Ruoff, R. S. Phys. Rev. Lett. 2000, 84, 5552-5555.

(24) Srivastava, D.; Menon, M.; Cho, K. Phys. Rev. Lett. 1999, 83, 2973-2976.

(25) Kis, A.; Zettl, A. Philos. Trans. R. Soc. A 2008, 366, 1591-1611.

(26) Wei, C.; Cho, K.; Srivastava, D. Phys. Rev. B 2003, 67, 115407.

(27) Yu, C.; Masarapu, C.; Rong, J.; Wei, B.; Jiang, H. Adv. Mater. 2009, 47, 4793-4797.

(28) Wang, C.; Cao, Q.; Ozel, T.; Gaur, A.; Rogers, J. A.; Shim, M. J. Am. Chem. Soc. 2005, 127, 11460-11468.

(29) Hobbie, E. K.; Simien, D. O.; Fagan, J. A.; Huh, J. Y.; Chung, J. Y.; Hudson, S. D.; Obrzut, J; Douglas, J. F.; Stafford, C. M. Phys. Rev. Lett. 2010, 104, 125505.

(30) Balberg, I.; Anderson, C. H.; Alexander, S.; Wagner, N. Phys Rev B 1984, 30, 3933-3943.

(31) Fagan, J. A.; Becker, M. L.; Chun, J.; Nie, P.; Bauer, B. J.; Simpson, J. R.; Hight Walker, A. R.; Hobbie, E. K. Langmuir 2008, 24, 13880-13889.

(32) Resasco, D. E.; Alvarez, W. E.; Pompeo, F.; Balzano, L.; Herrera, J. E.; Kitiyanan, B.; Borgna, A. J. Nanopart. Res. 2002, 4, 131-136.

(33) Cambré, S.; Wenseleers, W.; Goovaerts, E.; Resasco, D. E. ACS Nano 2010, 4, 6717-6724.

(34) Hobbie, E. K.; Fagan, J. A.; Becker, M. L.; Hudson, S. D.; Fakhri, N.; Pasquali, M. ACS Nano 2009, 3, 189-196.

(35) Mohraz, A.; Moler, D. B.; Ziff, R. M.; Solomon, M. J. Phys. Rev. Lett. 2004, 92, 155503.

(36) Lin-Gibson, S.; Schmidt, G.; Kim, H.; Han, C. C.; Hobbie, E. K. J. Chem. Phys. 2003, 119, 8080-8084.

(37) Stafford, C. M.; Harrison, C.; Beers, K. L.; Karim, A.; Amis, E. J.; Vanlandingham, M. R.; Kim, H. C.; Volksen, W.; Miller, R. D.; Simonyi, E. E. Nat. Mater. 2004, 3, 545-550.

(38) Jiang, H.; Khang, D.-Y.; Song, J.; Sun, Y.; Huang, Y.; Rogers, J. A. Proc. Natl. Acad. Sci. U. S. A. 2007, 104, 15607-15612.

(39) Hall, L. J.; Coluci, V. R.; Galvão, D. S.; Kozlov, M. E.; Zhang, M.; Dantas, S. O.; Baughman, R. H. Science 2009, 320, 504-507.

(40) Hobbie, E. K.; Fry, D. J. J. Chem. Phys. 2007, 126, 124907.

(41) Obrzut, J.; Douglas, J. F.; Kharchenko, S. B.; Migler, K. B. Phys. Rev. B 2007, 76, 195420.

(42) White, S. I.; DiDonna, B. A.; Mu, M.; Lubensky, T. C.; Winey, K. I. Phys. Rev. B 2009, 79, 024301.
(43) Simien, D.; Fagan, J. A.; Luo, W.; Douglas, J. F.; Migler, K.; Obrzut, J. ACS Nano 2008, 2, 1879-1884.

(44) Stafford, C. M.; Guo, S.; Harrison, C.; Chiang, M. Y. M. Rev. Sci. Instrum. 2005, 76, 062207.

(45) Hobbie, E. K.; Bauer, B. J.; Stephens, J.; Becker, M. L.; McGuiggan, P.; Hudson, S. D.; Wang, H. Langmuir 2005, 21, 10284-10287.

(46) Lee, J. N.; Park, C.; Whitesides, G. M. Anal. Chem. 2003, $75,6544-6554$. 